\title{
Expression of the NRF2 Target Gene NQO1 Is Enhanced in Mononuclear Cells in Human Chronic Kidney Disease
}

\author{
Jianlin Shen, ${ }^{1,2}$ Marianne Rasmussen, ${ }^{3}$ Qi-Rong Dong, ${ }^{1}$ Martin Tepel, ${ }^{2,3}$ and \\ Alexandra Scholze ${ }^{3,4}$ \\ ${ }^{1}$ Department of Orthopedics, The Second Affiliated Hospital of Soochow University, Suzhou, Jiangsu, China \\ ${ }^{2}$ Institute of Molecular Medicine, Cardiovascular and Renal Research, University of Southern Denmark, Odense, Denmark \\ ${ }^{3}$ Department of Nephrology, Odense University Hospital, Odense, Denmark \\ ${ }^{4}$ Institute of Clinical Research, University of Southern Denmark, Odense, Denmark
}

Correspondence should be addressed to Qi-Rong Dong; dqr@szgk.net and Alexandra Scholze; ascholze@health.sdu.dk

Received 7 April 2017; Revised 3 May 2017; Accepted 11 May 2017; Published 13 July 2017

Academic Editor: Ayman M. Mahmoud

Copyright (c) 2017 Jianlin Shen et al. This is an open access article distributed under the Creative Commons Attribution License, which permits unrestricted use, distribution, and reproduction in any medium, provided the original work is properly cited.

Reduced nuclear factor erythroid 2-related factor 2 (NRF2) pathway activity was reported in models of chronic kidney disease (CKD). Pharmacological activation of NRF2 is supposed to improve renal function, but data concerning the NRF2 activity in human $C K D$ are lacking. We investigated the NRF2 target $\mathrm{NAD}(\mathrm{P}) \mathrm{H}$ :quinone oxidoreductase 1 (NQO1) as a readout parameter for NRF2 activity in monocytes of CKD patients $(n=63)$ compared to those of healthy controls $(n=16)$. The NQO1 gene expression was quantified using real-time PCR and the protein content by in-cell Western assays. We found a 3-4-fold increase in NQO1 gene expression in CKD $1-5(n=29 ; 3.5$ for NQO1/ribosomal protein L41; $p<0.001)$. This was accompanied by a 1.1 -fold increase in NQO1 protein $(p=0.06)$. Cardiovascular disease prevalence was higher in CKD 1-5 patients with higher compared to those with lower NQO1 gene expression $(p=0.02)$. In advanced uremia, in dialysis patients $(n=34)$, NQO1 gene expression was less robustly upregulated than that in CKD 1-5, while NQO1 protein was not upregulated. We conclude that in mononuclear cells of CKD patients, the NRF2 pathway is activated by coexisting pathogenic mechanisms, but in advanced uremia, the effectiveness of this upregulation is reduced. Both processes could interfere with pharmacological NRF2 activation.

\section{Introduction}

The transcription factor NRF2 is a master transcriptional regulator of cellular response to oxidative and electrophilic stress. It activates a multitude of cellular defense processes through induction of its target genes, including drugmetabolizing and antioxidant enzyme genes [1]. Oxidative or electrophilic stress can activate the NRF2 pathway by an interaction with the cytoplasmic complex between Kelch-like ECH-associated protein 1 (KEAP1) and NRF2. As a result, NRF2 can escape ubiquitination and proteasomal degradation, accumulate, and translocate to the nucleus. It forms heterodimers with small Maf proteins, binds to antioxidant/electrophilic response elements, and thereby finally induces target gene expression $[1,2]$.
Cytosolic NQO1 is a conserved target gene of NRF2 and can serve to monitor the activity of the NRF2 pathway [2]. During its clinical development, the NRF2 activator bardoxolone methyl was investigated in a phase 1 clinical trial, in patients with advanced malignancies. In this trial, the NQO1 gene expression levels in peripheral blood mononuclear cells (PBMCs) were shown to be indicative of NRF2 pathway activation by the substance [3].

In animal models of renal diseases, impaired activity of the NRF2 pathway and downregulation of the NQO1 gene expression are major findings, both in renal and in nonrenal cells $[2,4,5]$. Furthermore, it was shown in animal models that activation of the NRF2 pathway and increased gene transcription, including those of NQO1, attenuated kidney injury [6-8]. Therefore, there is a strong interest 
in the therapeutic potential of NRF2 activators in kidney disease [9]. A phase $2 \mathrm{~b}$ clinical trial with the NRF2 activator bardoxolone methyl in CKD stages $3 \mathrm{~b}$ and 4 demonstrated an improvement in estimated glomerular filtration rate (eGFR) but suggested adverse effects, for example, on liver tissue [10]. A phase 3 clinical trial in CKD stage 4 patients (BEACON) with the same substance also showed an increased eGFR but had to be terminated because of a higher rate of cardiovascular events in the treatment group [11]. Currently, one more phase 2 clinical trial with bardoxolone methyl in CKD patients is recruiting participants (NCT02316821). In the publication of the so far largest clinical trial of bardoxolone methyl, the authors refer to the impairment of NRF2 activity-dependent gene transcription that was reported in animal models of CKD as one rational for the clinical application of the substance in CKD [11]. Accordingly, in our study, we investigated the gene expression of NQO1 as a parameter of NRF2dependent gene transcription in human CKD. We present a systematic analysis of this drug target on the level of gene expression and protein content in CKD patients with and without renal replacement therapy. The BEACON trial was terminated because of a higher rate of cardiovascular events in the intervention group [11]. A post hoc analysis by the manufacturer of the substance identified prior hospitalization for heart failure as a risk factor for heart failure experienced during bardoxolone treatment [12]. Therefore, we investigated a relation between the NQO1 gene expression as a measure for NRF2 pathway activity and cardiovascular disease (CVD) prevalence in patients with nondialysis-dependent CKD.

Since systemic NRF2 activators target the NRF2 pathway systemically and nonrenal adverse events were reported, both nonrenal and renal cells of CKD patients require investigation.

We therefore quantified the NQO1 gene expression as a readout parameter for NRF2 pathway activity in monocytes of patients with CKD with and without dialysis therapy and compared it to those of healthy control subjects. In parallel, NQO1 protein content was quantified in cells of the same subjects.

\section{Subjects and Methods}

2.1. Study Subjects. We prospectively enrolled 63 consecutive patients with CKD from the outpatient clinics of the Department of Nephrology, Odense University Hospital, and 16 healthy control subjects. Among the group of CKD patients, 34 were undergoing dialysis therapy (hemodialysis, $n=33$; peritoneal dialysis, $n=1$ ). The study was approved by the regional ethics committee and written informed consent was obtained. Hemodialysis patients were dialyzed with biocompatible membranes, three times per week, for 4-4.5 hours. Healthy control subjects were free from chronic or acute disease. Study participants were 30 years or older. CKD patients had verified CKD according to the 2012 Clinical Practice Guideline for the Evaluation and Management of Chronic Kidney Disease, and patients with dialysis therapy had been in dialysis treatment for at least 3 months
[13]. Exclusion criteria included pregnancy or breastfeeding, acute illness leading to hospital admission, and functioning renal allograft.

2.2. Collection of Clinical Data and Blood Samples. Clinical data were obtained by physical examination, medical history taking, and electronic medical records. This included the record of age, sex, height, weight, smoking status, medical history, and use of medications. Venous blood samples were drawn in the morning from study participants. Samples from hemodialysis patients were obtained before the start of hemodialysis sessions. Plasma and serum were separated and stored at $-80^{\circ} \mathrm{C}$. The eGFR was calculated using the 2009 CKD-EPI creatinine equation, and CKD stages were determined based on eGFR categories according to the KDIGO 2012 guideline [13].

2.3. Isolation of Circulating Monocytes. We isolated PBMCs by density gradient centrifugation (Histopaque-1077, Sigma-Aldrich, Denmark). Monocytes then were isolated from PBMCs using anti-CD14-coated superparamagnetic polystyrene beads (Fisher Scientific, Denmark) according to the manufacturer's protocol.

2.4. RNA Isolation, Reverse Transcription, and Quantitative Real-Time PCR (RT-qPCR). Total RNA was isolated from monocytes using an RNeasy Mini kit (Qiagen, Denmark) according to the protocol described by the manufacturer. cDNA was synthesized from $200 \mathrm{ng}$ of total RNA by reverse transcription using a Transcriptor First-Strand cDNA Synthesis Kit (Roche, Denmark). The amplification of genes was performed by quantitative real-time PCR using SYBR Green (FastStart Essential DNA Green Master, Roche, Denmark). The PCR conditions using a LightCycler96 Instrument (Roche, Denmark) were as follows: $95^{\circ} \mathrm{C}$ for $600 \mathrm{~s}$ and 50 cycles of $95^{\circ} \mathrm{C}$ for $10 \mathrm{~s}, 64^{\circ} \mathrm{C}$ (for NQO1, betaactin (ACTB), and ribosomal protein L41 (RPL41)) or $60^{\circ} \mathrm{C}$ (for TATA-box binding protein $(T B P)$ ) for $10 \mathrm{~s}$, and $72^{\circ} \mathrm{C}$ for $10 \mathrm{~s}$. The primers used were in Table 1 .

To ensure a high reliability of the gene expression results in our study, we tested a group of available reference genes in the cellular material of our study. We then employed the "NormFinder" algorithm (http://moma.dk/ normfinder-software; [14]). The reference genes with the highest expression stability in our sample material were RPL41, ACTB, and TBP with stability values (SE) of 0.12 (0.06), $0.18(0.06)$, and $0.22(0.05)$, respectively. We therefore used these three genes for the relative quantification of NQO1 gene expression. The ratio was calculated as follows: ratio $=2^{(\mathrm{Cq} \text { reference gene) }} / 2^{\text {(Cq NQO1) }}$.

PCR products were size-fractionated on agarose gels for product length control.

In the PCRs, water controls, no-template controls, and no-RT controls were included. A melting curve analysis was performed for each sample to ensure product homogeneity. All measurements were performed in duplicate.

2.5. SDS-PAGE and Western Blotting. Protein was extracted from monocytes using an extraction reagent for mammalian 
TABLE 1

\begin{tabular}{lrc}
\hline Name of gene product & $\begin{array}{c}\text { Forward primer } \\
\text { Reverse primer }\end{array}$ & Expected PCR product length (bp) \\
\hline NQO1 & 5'-CTGCCATCATGCCTGACTAA-3' & 216 \\
NM_000903.2 & 5'-TGCAGATGTACGGTGTGGAT-3' & \\
ACTB & 5'-GGACTTCGAGCAAGAGATGG-3' & 234 \\
NM_001101.3 & 5'-AGCACTGTGTTGGCGTACAG-3' & \\
RPL41 & 5'-AAGATGAGGCAGAGGTCC-3' & \\
NM_021104.1 & 5'-TCCAGAATGTCACAGGTCCA-3' & \\
TBP & 5'-TGCACAGGAGCCAAGAGTGAA-3' & \\
NM_003194.4 & 5'-CACATCACAGCTCCCCACCA-3' & 132 \\
\hline
\end{tabular}

cells including a protease inhibitor cocktail (cOmplete Lysis-M, pH 7.6, Roche, Denmark). Proteins were separated by $10 \%$ sodium-dodecyl-sulfate polyacrylamide gel electrophoresis (10\% RunBlue SDS gel, Expedeon, UK) at $120 \mathrm{~V}$ for 45 minutes and transferred to polyvinylidendifluoride membranes at $100 \mathrm{~V}$ for 75 minutes (Immun-Blot LF PVDF, Bio-Rad, USA). Membranes were blocked with blocking buffer (Superblock, Thermo Fisher Scientific, USA) for 1 hour at room temperature and incubated with the primary antibodies rabbit anti-human NQO1 (ab34173, Abcam, UK) or rabbit anti-human ACTB (sc-130656, Santa Cruz Biotechnology, Germany). After washing with tris(hydroxymethyl)aminomethane-buffered saline, the membranes were incubated with the fluorescence-labeled secondary antibody $\mathrm{F}\left(\mathrm{ab}^{\prime}\right)_{2}$-goat anti-rabbit IgG $(\mathrm{H}+\mathrm{L})$ Alexa Fluor 488 (Fisher Scientific, Denmark). Imaging was performed using a Carestream Imager 4000pro (Fisher Scientific, Denmark) at $535 \mathrm{~nm}$ emission with an excitation wavelength of $470 \mathrm{~nm}$.

2.6. Quantitative In-Cell Western Assay. To quantify the NQO1 protein content in circulating monocytes, in-cell Western assays were performed as recently described by our group $[15,16]$. For that purpose, monocytes were fixed with formaldehyde and permeabilized using Triton X-100 in 96 -well plates. Cells were blocked using $1 \%$ bovine serum albumin overnight at $4^{\circ} \mathrm{C}$, then incubated with the primary antibodies rabbit anti-human NQO1 (ab34173, Abcam, UK) or rabbit anti-human ACTB (sc-130656, Santa Cruz Biotechnology, Germany). After washing, cells were incubated with the fluorescence-labeled secondary antibody $\mathrm{F}\left(\mathrm{ab}^{\prime}\right)_{2}$-goat anti-rabbit IgG $(\mathrm{H}+\mathrm{L})$ Alexa Fluor 488 (Fisher Scientific, Denmark). All measurements were performed in triplicate, and the NQO1 protein content was analyzed relative to the ACTB protein content as an internal reference. Imaging was performed using an EnSpire Multimode Plate Reader (PerkinElmer, Denmark) at $520 \mathrm{~nm}$ emission with an excitation wavelength of $490 \mathrm{~nm}$.

\section{Statistics}

Continuous variables are given as median and interquartile range, and categorical variables are given as counts and percentages. Groups were compared using Kruskal-Wallis test with Dunn's posttest or Mann-Whitney test. Differences in categorical variables between groups were analyzed by $X^{2}$ test (GraphPad prism software, version 5.0, GraphPad Software, San Diego, CA). All statistical tests were twosided and a two-sided value of $p<0.05$ was considered statistically significant.

\section{Results}

Population characteristics of study subjects are shown in Table 2.

The NQO1 gene expression was significantly higher in monocytes from patients with CKD 1-5 (Kruskal-Wallis, $p=0.004$ for NQO1/ACTB; $p<0.001$ for NQO1/RPL41; and $p<0.001$ for NQO1/TBP; Figures 1(a) and 1 (b)). Compared to healthy controls, patients with CKD 1-5 showed a 3-4-fold increase in the NQO1 gene expression (3.1 for NQO1/ACTB, 3.5 for NQO1/RPL41, and 4.2 for NQO1/TBP). In CKD 5 patients undergoing dialysis therapy (CKD 5D), median NQO1 gene expression was numerically higher than that in healthy controls but this was significant only for the NQO1/ $T B P$ ratio (a 2.6-fold increase for $N Q O 1 / T B P, p<0.05$, Figure 1(b)). The relation between the NQO1 gene expression level and CKD stage followed approximately a bell shape. This is shown in the Supplementary Figure 1 available online at https://doi.org/10.1155/2017/9091879 representing the distribution of the NQO1 gene expression at the different CKD stages including advanced CKD with hemodialysis therapy (Kruskal-Wallis, $p=0.005$ ).

We also quantified the NQO1 protein content in cells of the identical blood samples used for the gene expression analyses described above. First, we proved that in the cell material used for our study, the antibodies effectively detected NQO1 and ACTB proteins. Immunoblot analyses showed staining of protein bands at the expected sizes (Figure 2(a)). Figure 2(b) shows a representative example of an in-cell Western assay used for the relative quantification of the NQO1 protein content in monocytes. Measurements were performed in triplicate for each subject. Figure 2(c) depicts the summary data for all in-cell Western analyses. The NOQ1 protein content showed a 1.1-fold increase in CKD 1-5 patients compared to healthy controls (Mann-Whitney, $p=0.06)$. In CKD 5D, the NQO1 protein content was not different from control subjects (Mann-Whitney, $p=0.72$ ).

Furthermore, since cardiovascular events led to the early termination of a large NRF2 activator trial in 
TABLE 2: Clinical and demographical population characteristics.

\begin{tabular}{|c|c|c|c|}
\hline & Healthy $(n=16)$ & CKD 1-5 $(n=29)$ & CKD 5D $(n=34)$ \\
\hline Age, years & $38(33-48)$ & $68(58-77)$ & $67(55-76)$ \\
\hline Men, $n(\%)$ & $9(56)$ & $19(66)$ & $23(68)$ \\
\hline BMI, $\mathrm{kg} / \mathrm{m}^{2}$ & $24(22-26)$ & $30(24-37)^{\mathrm{a}}$ & $24(22-28)^{\mathrm{b}}$ \\
\hline Smoking, $n(\%)$ & $2(13)$ & $3(10)$ & $6(18)$ \\
\hline Kidney disease, underlying cause, $n(\%)$ & None & & \\
\hline Glomerulonephritis & & $8(28)$ & $5(15)$ \\
\hline Hypertensive nephropathy & & $3(10)$ & $5(15)$ \\
\hline Interstitial nephritis & & $2(7)$ & $4(12)$ \\
\hline Diabetic nephropathy & & $1(3)$ & $2(6)$ \\
\hline Hereditary kidney disease & & $1(3)$ & $2(6)$ \\
\hline Other/unknown & & $14(48)$ & $16(47)$ \\
\hline Comorbidities, $n(\%)$ & None & & \\
\hline Hypertension & & $28(97)$ & $26(76)$ \\
\hline Diabetes & & $8(28)$ & $8(24)$ \\
\hline \multicolumn{4}{|l|}{ CVD } \\
\hline Myocardial infarction, coronary artery disease & & $6(21)$ & $10(29)$ \\
\hline Heart failure & & $8(28)$ & $16(47)$ \\
\hline Cerebrovascular disease & & $4(14)$ & $6(18)$ \\
\hline Peripheral artery disease & & $1(3)$ & $9(26)$ \\
\hline Medications & None & & \\
\hline AT receptor antagonist, ACE inhibitor & & $22(76)$ & $16(47)$ \\
\hline Beta blocker & & $9(31)$ & $15(44)$ \\
\hline Calcium channel inhibitors & & $13(45)$ & $9(26)$ \\
\hline Platelet aggregation inhibitor & & $6(21)$ & $6(18)$ \\
\hline Diuretic & & $19(66)$ & $11(32)$ \\
\hline Erythropoietin analog & & $6(21)$ & $29(85)$ \\
\hline Coumarin derivatives & & $5(17)$ & $6(18)$ \\
\hline $\mathrm{eGFR}, \mathrm{mL} / \mathrm{min} / 1.73 \mathrm{~m}^{2}$ & n.d. & $28(17-43)$ & n.a. \\
\hline Time on dialysis, months & n.a. & n.a. & $22(11-49)$ \\
\hline $\mathrm{CRP}, \mathrm{mg} / \mathrm{L}$ & n.d. & $5.3(2.3-10.8)^{\mathrm{c}}$ & $3.0(1.4-10.5)^{\mathrm{d}}$ \\
\hline Albumin, g/L & n.d. & $38(35-41)^{\mathrm{e}}$ & $38(36-40)$ \\
\hline
\end{tabular}

Values are given as median (25\%-75\% percentile) or number (percentage). BMI: body mass index; AT: angiotensin; ACE: angiotensin converting enzyme; CRP: C-reactive protein; n.d.: not done; n.a.: not applicable. ${ }^{\mathrm{a}} n=27 ;{ }^{\mathrm{b}} n=33 ;{ }^{\mathrm{c}} n=28 ;{ }^{\mathrm{d}} n=33 ;{ }^{\mathrm{e}} n=28$.

non-hemodialysis-dependent CKD, we investigated the prevalence of CVD in our study. For the analysis of the NQO1 expression and prevalence of CVD in nonhemodialysis-dependent CKD stages $1-5$, we grouped the patients according to their NQO1 gene expression below or above the median and compared the frequency of CVD between them. When we analyzed the overall prevalence of CVD (myocardial infarction/coronary artery disease, heart failure, cerebrovascular disease, and peripheral artery disease), it was 2 out of 14 in the patient group below median gene expression whereas in the group above median NQO1 gene expression, the prevalence of CVD was 8 out of 14 ( $X^{2}$ test, $\left.p=0.02\right)$. The only common underlying single disease type in our patient group was cardiac insufficiency ( 1 out of 14 in the below median group versus 7 out of 14 in the above median group; $X^{2}$ test, $p=0.18)$.

\section{Discussion}

This study showed for mononuclear cells of CKD patients (i) increased gene expression of the NRF2 target NQO1 in CKD $1-5$, (ii) compared to CKD $1-5$ a less robust increase in the NQO1 gene expression in CKD 5D, (iii) slightly increased NQO1 protein content in $\mathrm{CKD} 1-5$, and (iv) higher prevalence of CVD among CKD 1-5 patients with a higher NQO1 gene expression.

In our study, we investigated monocytes from peripheral blood of CKD patients. The monocyte-macrophage lineage is of special importance in CKD. On the one hand, they are involved in kidney injury and fibrosis [17, 18]. On the other hand, monocytes and monocyte-derived macrophages are involved in the pathogenesis of atherosclerosis and vascular disease in CKD [19-22]. The latter is important with respect to our finding of higher CVD 

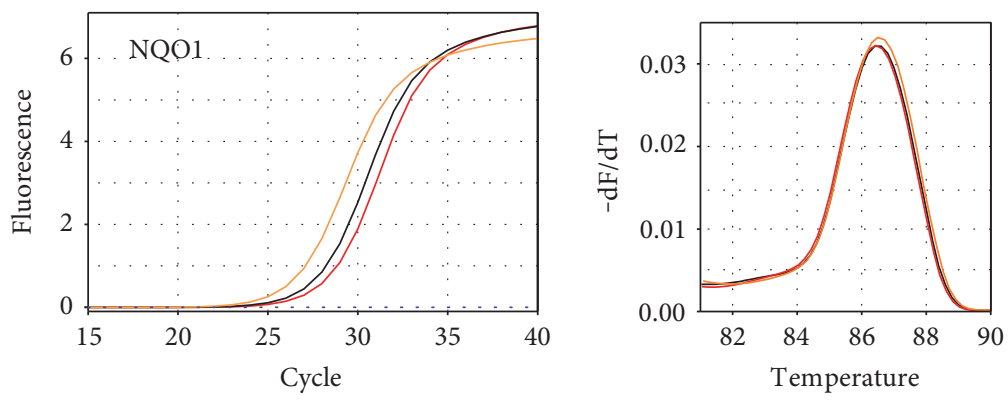

(a)
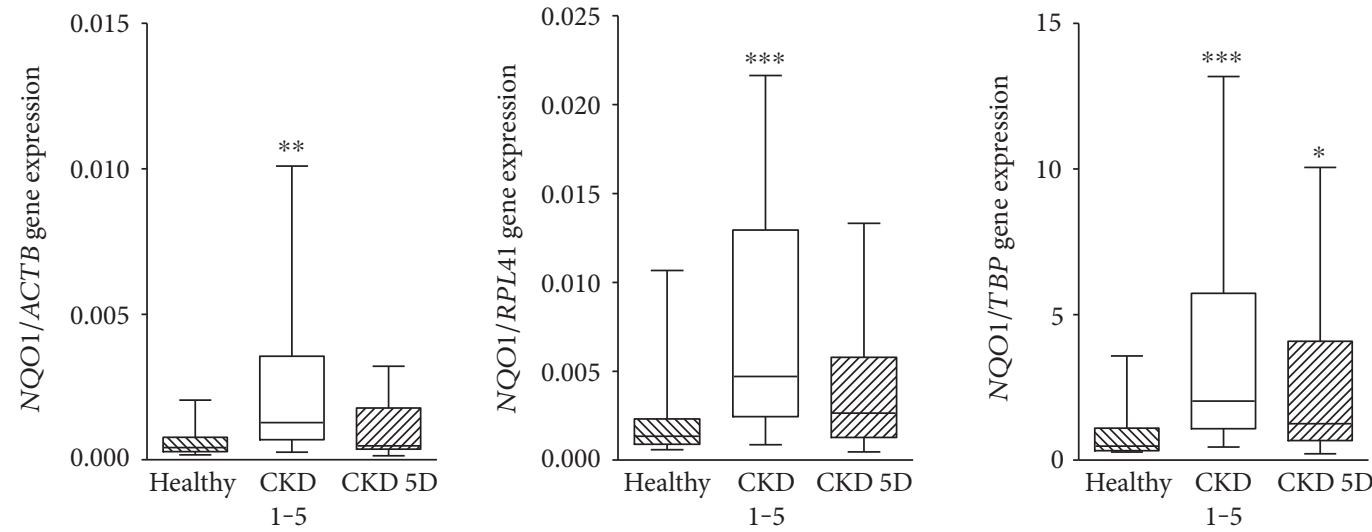

(b)

FIGURE 1: NQO1 gene expression. (a) Amplification curves and melting curves for NQO1 in monocytes from a patient with CKD 4 (CKD, orange), a hemodialysis patient (CKD 5D, black), and a healthy subject (red). (b) Box and whisker plots (whiskers, minimum to maximum) showing summary data of the NQO1 gene expression in healthy subjects $(n=16)$, CKD $1-5$ patients $(n=29)$, and CKD 5D patients $(n=34)$ normalized to $A C T B, R P L 41$, and TBP. Comparison by Dunn's posttest. ${ }^{*} p<0.05$; ${ }^{* *} p<0.01$; ${ }^{* * *} p<0.001$.

prevalence in CKD patients with an NQO1 gene expression above the median in monocytes.

Why did we choose NQO1 and in particular the NQO1 gene expression as a readout parameter for NRF2 pathway activity and activation in kidney disease? Several lines of evidence support our approach. First, NQO1 belongs to the conserved NRF2 target genes, and in a recent integrated transcriptomic and proteomic analysis of NRF2 function, NQO1 was confirmed as a robust marker for NRF2 activity $[2,23]$. Second, reduced expression of NQO1 was shown in several kidney disease models, also in nonrenal cells, and upregulation of NQO1 was repeatedly shown in NRF2dependent kidney protection, again including nonrenal cells $[4-8,24]$. Third, it has been shown that the NQO1 expression is stimulated by the NRF2 activators CDDO (1-[2-cyano3-,12-dioxooleana-1,9(11)-dien-28-oyl]imidazolide), dh404 (2-cyano-3,12-dioxooleana-1,9-dien-28-oic acid-9,11-dihydro-trifluoroethyl amide), and bardoxolone methyl and that this effect is abrogated in $N R F 2^{-/-}$mice $[5,8,23]$. Notably, bardoxolone methyl stimulates the NQO1 gene expression also in PBMCs [3].

Some consideration should be given to the effect size of the NQO1 increase in our study. With respect to the gene expression of NQO1, we confirmed a 3-4-fold increase in NQO1 mRNA in CKD 1-5 patients compared to healthy control subjects using three different housekeeping genes for the relative quantification. A 5.6-fold increase in NQO1 mRNA was reported to be present in PBMCs from cancer patients after 3 weeks of treatment with bardoxolone methyl [3]. In T cell-specific KEAP1-deficient mice, $\mathrm{T}$ cells showed an 8-fold relative change in NQO1 mRNA [7]. In a mouse model of ischemia-reperfusion injury- (IRI-) induced kidney damage, the maximal response of NQO1 mRNA to IRI was an $~ 1.8$-fold increase in wild-type mice and a 5-fold increase in KEAP1-knockdown mice [8]. We therefore conclude that the difference in NQO1 mRNA level that we observed in our study is of relevant magnitude and points to an activation of the NRF2 pathway in CKD patients per se.

On the protein level, the NQO1 protein content in CKD 1-5 patients was not significantly increased to 1.1 -fold compared to that in control subjects in our study. Similarly, the treatment of mice with bardoxolone methyl resulted in an $\sim 1.3$-fold increase in NQO1 protein level [23]. Treatment of $5 / 6$ nephrectomy rats with dh404 resulted in an $\sim 3.3$-fold increase in NQO1 protein in the colon [5]. However, the increase in the NQO1 protein content that we observed in CKD 1-5 needs further investigation.

Effects on gene expression and protein content in $\mathrm{CKD}$ are mediated by a multitude of mechanisms. The observed increase in the NQO1 gene expression in our study might be a response to NRF2-stimulating conditions prevalent in patients with $\mathrm{CKD}$, like oxidative stress or 

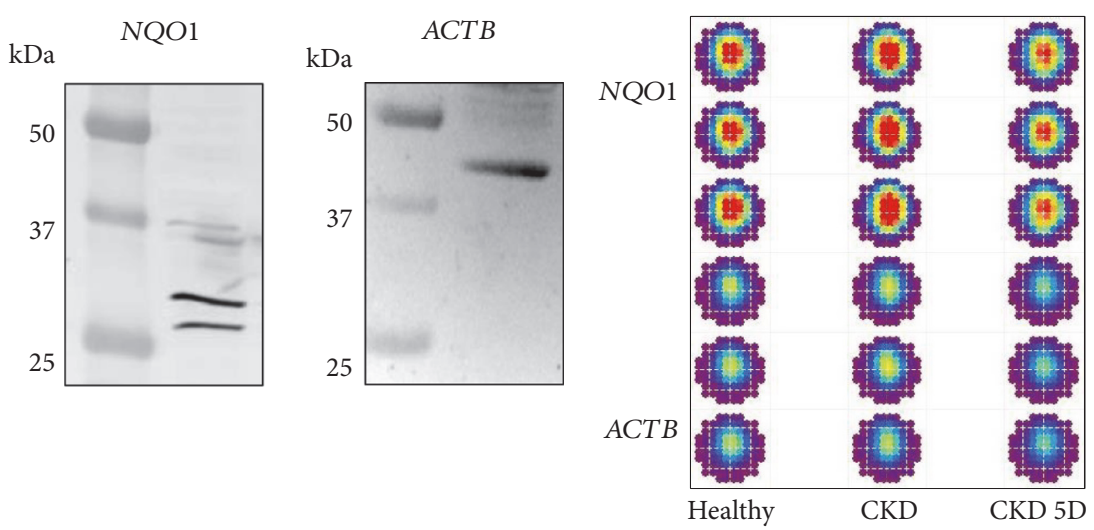

(a)

(b)

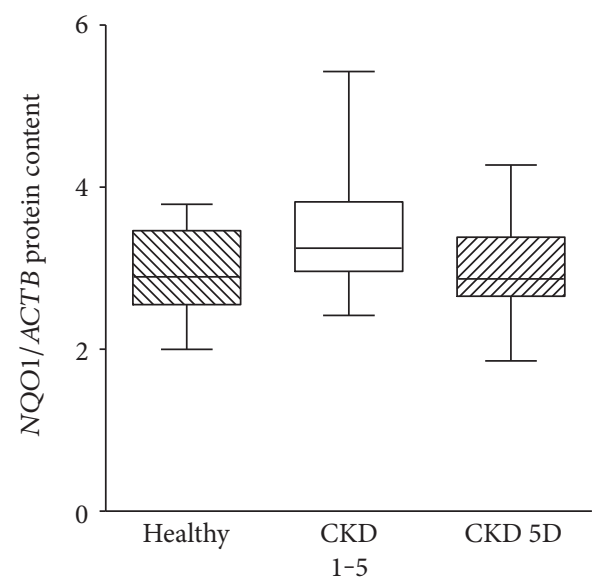

(c)

FIGURE 2: NQO1 protein content. (a) To show the effective detection of NQO1 and ACTB protein by antibodies in the cell material used in our study, we performed immunoblot analyses with cells obtained from healthy control subjects. Immunoblots of NQO1 (expected size 26/27 kDa and $31 \mathrm{kDa}$ ) and $A C T B$ (expected size $42 \mathrm{kDa}$ ) in monocytes are shown. (b) Pseudocolored fluorescence intensities of in-cell Western assays for the quantification of the NQO1 protein content relative to the ACTB protein content in monocytes from a healthy subject, a patient with $\mathrm{CKD}$, and a patient with CKD 5D. Measurements were always performed in triplicate. (c) Box and whisker plots (whiskers, minimum to maximum) showing summary data of NQO1 protein in healthy subjects $(n=13)$, CKD $1-5$ patients $(n=23)$, and CKD 5D patients $(n=29)$ normalized to ACTB. $p=0.07$ by Kruskal-Wallis test.

lipopolysaccharide-induced inflammation [25, 26]. Patients with coexisting CVD, which is also associated with oxidative stress, might be especially affected [27]. This is supported by the higher CVD prevalence in CKD 1-5 patients with a higher NQO1 gene expression that we observed. In addition, low concentrations of the uremic toxin methylglyoxal were shown to increase the NQO1 mRNA concentration [28].

We also observed that the increase in the NQO1 gene expression in $\mathrm{CKD} 5 \mathrm{D}$ patients was less pronounced compared to that in CKD 1-5 patients. This is in line with a depressive effect of high concentrations of uremic toxins on the NRF2 pathway. This effect might be more pronounced in this patient group with advanced uremia. For example, the uremic toxin indoxyl sulfate was shown to downregulate NRF2 mRNA and NRF2 protein content [24].

The protein content of NQO1 appeared slightly upregulated in CKD 1-5 patients and not upregulated in long-term uremic CKD 5D patients. Such discrepancy between gene expression and protein content was already demonstrated by our group for another cytosolic antioxidant protein that also is under the transcriptional regulation of an antioxidant response element-superoxide dismutase type 1 [15].

Our study adds important information to the discussion about the putative use of NRF2 activators in CKD. NRF2 activators serve to upregulate the expression of NRF2 target genes like NQO1. However, our study showed that the NQO1 gene expression was already significantly upregulated in monocytes, which points to a relevant stimulation of the NRF2 pathway in these cells in CKD patients. Therefore, as the systemic application of NRF2 activators affects different cell and tissue types, the NRF2 pathway in kidney disease needs to be investigated in different human cells and tissues. This is of relevance as the overactivation of a potent transcription factor like NRF2 was suggested as potentially deleterious for the cardiovascular system [27]. Therefore, a preexisting activation of the NRF2 system in CKD patients especially with CVD, as suggested by our results, could be seen in line with the increased rate of cardiovascular events in the BEACON trial [11]. Moreover, an additional 
depressive effect of CKD on antioxidant enzymes, especially in advanced uremic conditions, could reduce effectiveness of NRF2 activation.

Taken together, we showed a relevant upregulation in gene expression of the NRF2 target NQO1 in patients with CKD 1-5 together with a slight increase in the NQO1 protein content in monocytes from these patients. Moreover, we found that in more pronounced uremia (CKD 5D), the NQO1 gene expression was less upregulated than that in CKD 1-5 and NQO1 protein content was not increased. We conclude that in CKD patients, NRF2 activation is modulated through influence on both gene expression and protein content of NRF2 targets in a complex way.

\section{Conflicts of Interest}

None are declared.

\section{Acknowledgments}

The study was partly supported by the Second Affiliated Hospital of Soochow University Preponderant Clinic Discipline Group project funding (XKQ2015003) and National Natural Science Foundation of China (81171730 to Qi-Rong Dong).

\section{References}

[1] T. Suzuki and M. Yamamoto, "Molecular basis of the Keap1NRF2 system," Free Radical Biology and Medicine, vol. 88, Part B, pp. 93-100, 2015.

[2] F. E. Mutter, B. K. Park, and I. M. Copple, "Value of monitoring NRF2 activity for the detection of chemical and oxidative stress," Biochemical Society Transactions, vol. 43, no. 4, pp. 657-662, 2015.

[3] D. S. Hong, R. Kurzrock, J. G. Supko et al., "A phase I first-inhuman trial of bardoxolone methyl in patients with advanced solid tumors and lymphomas," Clinical Cancer Research, vol. 18, no. 12, pp. 3396-3406, 2012.

[4] H. J. Kim and N. D. Vaziri, "Contribution of impaired NRF2Keap1 pathway to oxidative stress and inflammation in chronic renal failure," American Journal of Physiology. Renal Physiology, vol. 298, no. 3, pp. F662-F671, 2010.

[5] W. L. Lau, S. M. Liu, S. Pahlevan et al., "Role of NRF2 dysfunction in uremia-associated intestinal inflammation and epithelial barrier disruption," Digestive Diseases and Sciences, vol. 60, no. 5, pp. 1215-1222, 2015.

[6] Y. Miyazaki, A. Shimizu, I. Pastan et al., "Keap1 inhibition attenuates glomerulosclerosis," Nephrology, Dialysis, Transplantation, vol. 29, no. 4, pp. 783-791, 2014.

[7] S. Noel, M. N. Martina, S. Bandapalle et al., "T lymphocytespecific activation of NRF2 protects from AKI," Journal of the American Society of Nephrology, vol. 26, no. 12, pp. 29893000, 2015.

[8] M. Nezu, T. Souma, L. Yu et al., "Transcription factor NRF2 hyperactivation in early-phase renal ischemia-reperfusion injury prevents tubular damage progression," Kidney International, vol. 91, no. 2, pp. 387-401, 2017.

[9] S. Noel, A. R. Hamad, and H. Rabb, "Reviving the promise of transcription factor NRF2-based therapeutics for kidney diseases," Kidney International, vol. 88, no. 6, pp. 1217-1218, 2015.
[10] P. E. Pergola, P. Raskin, R. D. Toto et al., "Bardoxolone methyl and kidney function in CKD with type 2 diabetes," The New England Journal of Medicine, vol. 365, no. 4, pp. 327-336, 2011.

[11] D. de Zeeuw, T. Akizawa, P. Audhya et al., "Bardoxolone methyl in type 2 diabetes and stage 4 chronic kidney disease," The New England Journal of Medicine, vol. 369, no. 26, pp. 2492-2503, 2013.

[12] M. P. Chin, D. Wrolstad, G. L. Bakris et al., "Risk factors for heart failure in patients with type 2 diabetes mellitus and stage 4 chronic kidney disease treated with bardoxolone methyl," Journal of Cardiac Failure, vol. 20, no. 12, pp. 953-958, 2014.

[13] Kidney Disease: Improving Global Outcomes (KDIGO) CKD Work Group, "KDIGO 2012 clinical practice guideline for the evaluation and management of chronic kidney disease," Kidney International, vol. 3, no. 1, Supplement 2013, pp. 1-150, 2013.

[14] C. L. Andersen, J. L. Jensen, and T. F. Ørntoft, "Normalization of real-time quantitative reverse transcription-PCR data: a model-based variance estimation approach to identify genes suited for normalization, applied to bladder and colon cancer data sets," Cancer Research, vol. 64, no. 15, pp. 5245-5250, 2004.

[15] A. Scholze, K. Krueger, M. Diedrich et al., "Superoxide dismutase type 1 in monocytes of chronic kidney disease patients," Amino Acids, vol. 41, no. 2, pp. 427-438, 2011.

[16] K. Krueger, J. Shen, A. Maier, M. Tepel, and A. Scholze, "Lower superoxide dismutase 2 (SOD2) protein content in mononuclear cells is associated with better survival in patients with hemodialysis therapy," Oxidative Medicine and Cellular Longevity, vol. 2016, Article ID 7423249, 8 pages, 2016.

[17] Q. Cao, D. C. Harris, and Y. Wang, "Macrophages in kidney injury, inflammation, and fibrosis," Physiology (Bethesda), vol. 30, no. 3, pp. 183-194, 2015.

[18] H. Han, J. Zhu, Y. Wang et al., "Renal recruitment of B lymphocytes exacerbates tubulointerstitial fibrosis by promoting monocyte mobilization and infiltration after unilateral ureteral obstruction," The Journal of Pathology, vol. 241, no. 1, pp. 80 90, 2017.

[19] T. Watanabe, K. Yasunari, M. Nakamura, and K. Maeda, "Carotid artery intima-media thickness and reactive oxygen species formation by monocytes in hypertensive patients," Journal of Human Hypertension, vol. 20, no. 5, pp. 336-340, 2006.

[20] C. Ulrich, G. H. Heine, M. K. Gerhart, H. Köhler, and M. Girndt, "Proinflammatory CD14+CD16+ monocytes are associated with subclinical atherosclerosis in renal transplant patients," American Journal of Transplantation, vol. 8, no. 1, pp. 103-110, 2008.

[21] V. Kon, M. F. Linton, and S. Fazio, "Atherosclerosis in chronic kidney disease: the role of macrophages," Nature Reviews. Nephrology, vol. 7, no. 1, pp. 45-54, 2011.

[22] S. Zewinger, T. Schumann, D. Fliser, and T. Speer, "Innate immunity in CKD-associated vascular diseases," Nephrology, Dialysis, Transplantation, vol. 31, no. 11, pp. 1813-1821, 2016.

[23] L. M. Shelton, A. Lister, J. Walsh et al., "Integrated transcriptomic and proteomic analyses uncover regulatory roles of NRF2 in the kidney," Kidney International, vol. 88, no. 6, pp. 1261-1273, 2015.

[24] D. Bolati, H. Shimizu, M. Yisireyili, F. Nishijima, and T. Niwa, "Indoxyl sulfate, a uremic toxin, downregulates renal 
expression of NRF2 through activation of NF- $\kappa \mathrm{B}$," $B M C$ Nephrology, vol. 14, p. 56, 2013.

[25] T. Nguyen, P. Nioi, and C. B. Pickett, "The NRF2-antioxidant response element signaling pathway and its activation by oxidative stress," The Journal of Biological Chemistry, vol. 284, no. 20, pp. 13291-13295, 2009.

[26] H. Song, Y. Lu, Z. Qu et al., "Effects of aged garlic extract and FruArg on gene expression and signaling pathways in lipopolysaccharide-activated microglial cells," Scientific Reports, vol. 6, article 35323, 2016.

[27] P. Jakobs, V. Serbulea, N. Leitinger, A. Eckers, and J. Haendeler, "Nuclear factor (erythroid-derived 2)-like 2 and thioredoxin-1 in atherosclerosis and ischemia/reperfusion injury in the heart," Antioxidants \& Redox Signaling, vol. 26, no. 12, pp. 630-644, 2017.

[28] D. Li, S. Ma, and E. M. Ellis, "NRF2-mediated adaptive response to methyl glyoxal in HepG2 cells involves the induction of AKR7A2," Chemico-Biological Interactions, vol. 234, pp. 366-371, 2015. 


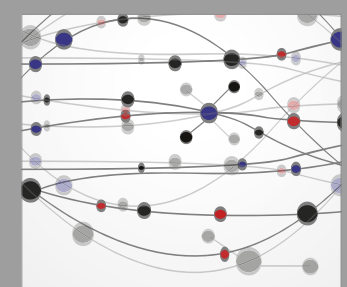

The Scientific World Journal
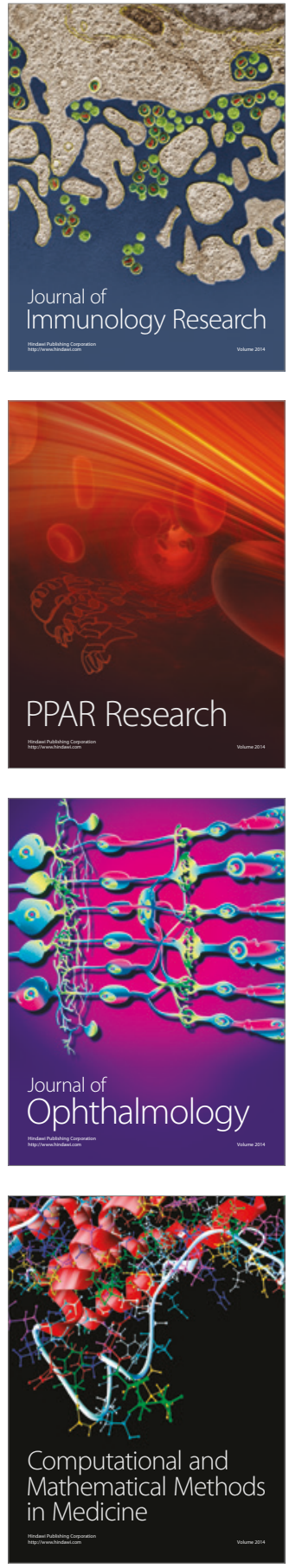

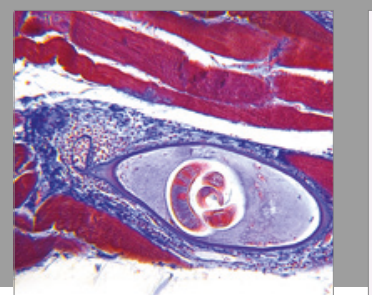

Gastroenterology Research and Practice
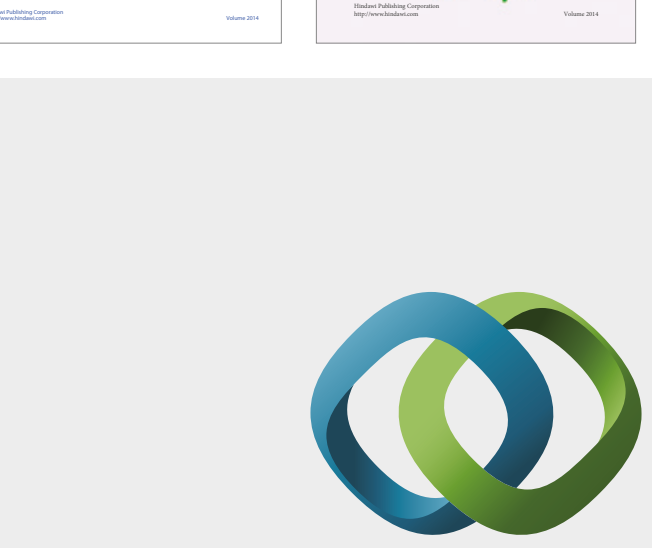

\section{Hindawi}

Submit your manuscripts at

https://www.hindawi.com
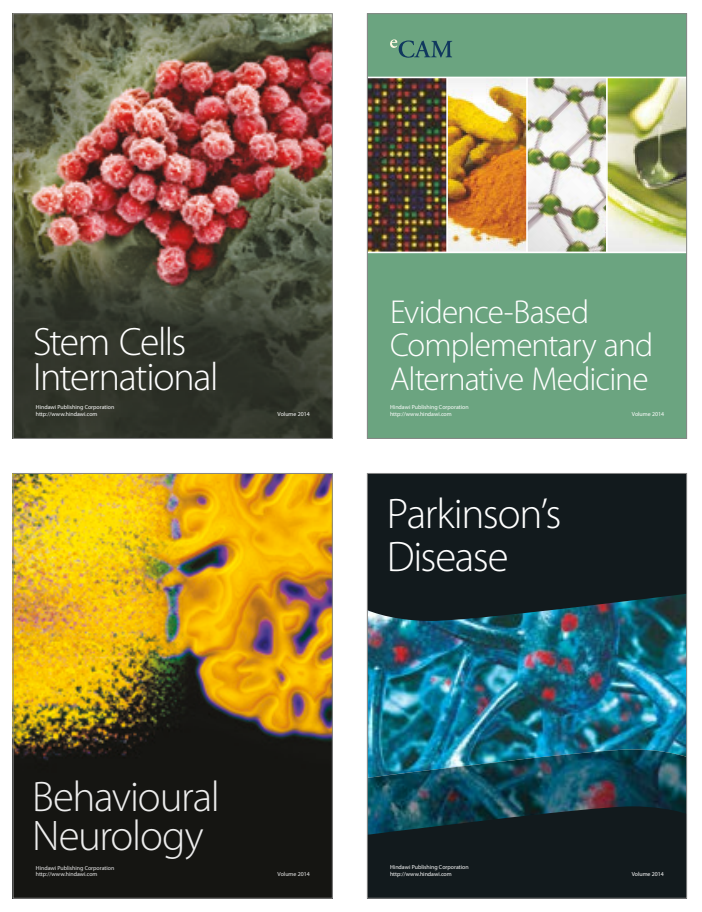
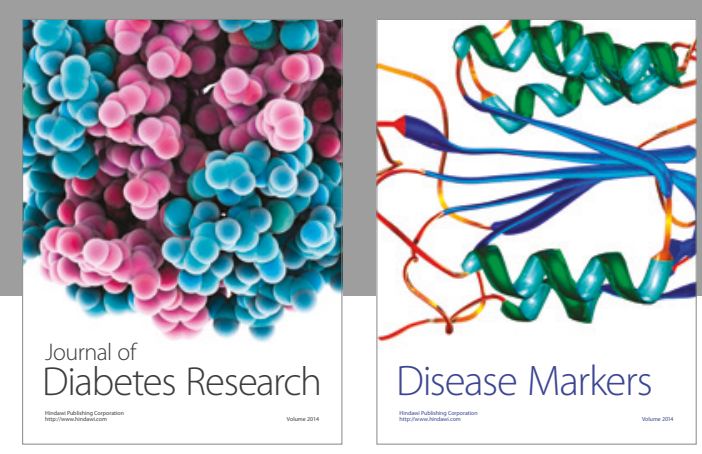

Disease Markers
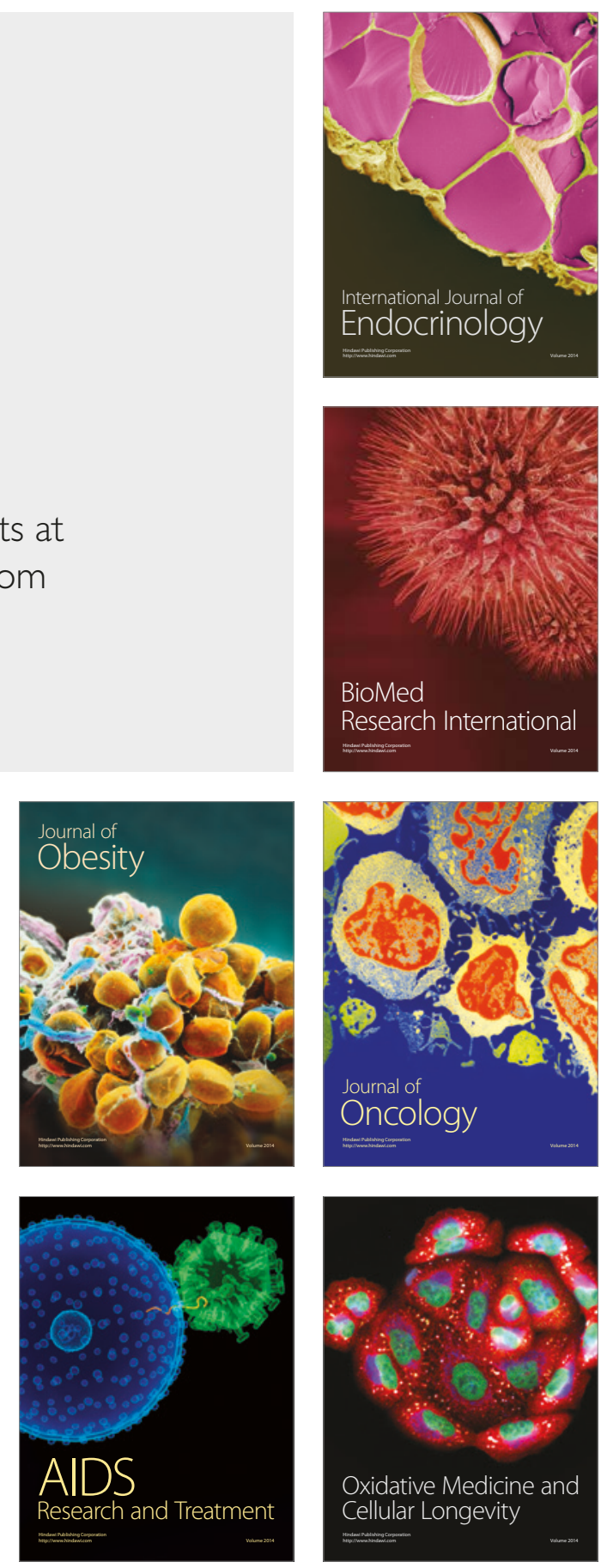